Multivariate Behavioral Research, 39 (2), 203-230

Copyright @ 2004, Lawrence Erlbaum Associates, Inc.

\title{
On the Relationship Between Circumplexes: Affect and Wiggins' IAS
}

\author{
Michelle S. M. Yik \\ Hong Kong University of Science and Technology \\ James A. Russell \\ Boston College
}

This article offers a new method to describe the relationship between two circumplexes, illustrated empirically with Wiggins' (1995) Interpersonal Adjective Scales (IAS) and Yik, Russell, and Steiger's (2004) 12-Point Affect Circumplex Scales (12-PACS). Michael Browne's CIRCUM-extension procedure was used to place each circumplex within the other. Simulations showed this procedure can reveal the number of dimensions of overlap between the two and can estimate the magnitude and location of the overlap. The IAS space overlaps the 12-PACS space on one axis, which falls at $35^{\circ}$ within the 12-PACS space (pleasant activated versus unpleasant deactivated) and at $71^{\circ}$ within the IAS space, which is close to where Extraversion (Factor I of the Five Factor Model) also lies.

Since the introduction of the circumplex by Guttman in 1954, a circular ordering of variables has proved useful in a variety of domains (Fabrigar, Visser, \& Browne, 1997; Plutchik \& Conte, 1997). Examples include interpersonal behaviors (Leary, 1957), interpersonal dispositions (Wiggins, 1979), emotions (Russell, 1980), vocational interests (Holland, 1973), interpersonal problems (Alden, Wiggins, \& Pincus, 1990), psychopathology (Widiger, Trull, Clarkin, Sanderson, \& Costa, 1994), parent-child relationships (Schaefer, 1959), and color perception (Shepard, 1962). Techniques for

Jerry Wiggins is our mentor, friend, and inspiration, and we feel a deep honor in being able to contribute to this special issue celebrating his career. Jerry-many, many thanks for everything, but especially for your elegant style pervasive in your theories, writing, teaching, and life.

We profusely thank Michael Browne for his advice on statistical matters. We also thank Martin Carroll, Ben Chan, Steven So, and Ellick Wong for their help in preparing the manuscript. Finally, we thank Alan Jin and Jianping Wang for preparing the figures.

Preparation of this article was facilitated by a RGC Direct Allocation Grant (DAG02/ 03.HSS14) awarded to Michelle Yik.

Correspondence can be directed to Michelle Yik, Hong Kong University of Science and Technology, Division of Social Science, Clear Water Bay, Kowloon, Hong Kong. Electronic mail: myik@ust.hk. Fax: (852) 2335-0014. 
testing circumplexity within a domain have evolved from visual inspection (looking for a cosine pattern of correlations ${ }^{1}$ ) to Browne's (1992) structural equation modeling procedure (Tracey, 2000). The purpose of the present article is to help propel a similar evolution in describing the relationship between circumplexes. We propose a method for this purpose and illustrate our technique empirically with two known circumplexes: Wiggins' (1995) Interpersonal Adjective Scales (IAS) and Yik et al.'s (2004) 12-Point Affect Circumplex Scales (12-PACS).

\section{Interpersonal Dispositions}

Based on seminal work by Sullivan (1953) and Leary (1957), and later work by Lorr and McNair (1965), Benjamin (1974), and Kiesler (1983), Wiggins and his colleagues built a descriptive model and accompanying scales (IAS) for interpersonal behaviors and dispositions. Developed from the universe of interpersonal adjectives contained in Goldberg's (1977) master pool, the IAS underwent extensive development, with the final model in the form of a circumplex (Wiggins, 1995). An early version of the scales consisted of 16 facets, named and ordered alphabetically (A through P). In a later version, adjacent facets were combined, resulting in eight octants, each coded by a two-letter combination (e.g., PA, BC), arranged in a circular order. With the early methods of testing circumplexity, the IAS repeatedly appeared to be a circumplex (Kiesler, 1996; Tracey \& Schneider, 1995; Wiggins, 1995). However, when Gaines et al. (1997) examined the IAS with general structural equation modeling, they concluded that it does not conform to an ideal circumplex. Gurtman and Pincus (2000) then used Browne's (1992) CIRCUM, a structural equation modeling approach specifically designed to test circumplexity, and showed that the IAS excel in the structural requirements of a circumplex model. The current study provides an opportunity to confirm Gurtman and Pincus's results.

Another purpose was to extend the IAS' nomological network by examining its lawful relations to yet another important domain, affect. The IAS have been found to be embedded in a network of constructs, such as interpersonal problems (Alden, Wiggins, \& Pincus, 1990), personality disorders (Wiggins \& Pincus, 1989), mate selection (Buss \& Barnes, 1986), manipulation tactics (Buss, 1989), and nonverbal behaviors (Gifford, 1991), to name a few. Wiggins himself followed Foa and Foa's (1974) theory in emphasizing dominance and love as the underlying processes defining the

\footnotetext{
${ }^{1} \mathrm{~A}$ cosine function is just a sine function shifted $90^{\circ}$. It is deemed more appropriate to describe a correlation function because the cosine function of $0^{\circ}$ is 1.00 (the correlation coefficient of a variable with itself is 1.00 ) whereas the sine function of $0^{\circ}$ is 0.00 .
} 
eight IAS variables. Dominance and love thus provide a specific rotation of the two-dimensional space. An alternative rotation of the axes yields Extraversion and Agreeableness of the Five Factor Model (McCrae \& Costa, 1989; Pincus, 2002; Trapnell \& Wiggins, 1990). ${ }^{2}$ As McCrae and Costa (1989) pointed out, "The five-factor model provides a larger framework in which to orient and interpret the circumplex, and the interpersonal circle provides a useful elaboration about aspects of two of the five factors" (p. 593). Examining the relation of the IAS to affect provides another datum on the question of rotation.

\section{Momentary Affect}

Various dimensional models have been proposed to characterize the covariations of self-reported momentary affective feelings. Major models include Russell's (1980) circumplex, Thayer's (1996) energetic and tense arousal, Larsen and Diener's (1992) eight combinations of pleasantness and activation, and Watson and Tellegen's (1985) positive and negative affect. All four models have been shown to fit comfortably within the same twodimensional affective space defined by the bipolar axes of pleasure and arousal (Yik, Russell, \& Feldman Barrett, 1999). This integrated model shows cross-language generalizability (Russell, 1983; Russell, Lewicka, \& Niit, 1989; Yik \& Russell, 2001, 2003; Yik, Russell, \& Ahn, 2003; Yik, Russell, Oceja, \& Fernández Dols, 2000; Yik, Russell, \& Suzuki, 2003) with replications in Chinese, Croatian, Estonian, Greek, Gujarti, Japanese, Korean, Polish, and Spanish. Watson and Tellegen's (1985) Positive Affect and Negative Affect structure was replicated in Japanese (Watson, Clark, \& Tellegen, 1984), Hebrew (Almagor \& Ben-Porath, 1989), Castillian Spanish (Joiner, Sandín, Chorot, Lostao, \& Marguina, 1997), and Tagalog (Church, Katigbak, Reyes, \& Jensen, 1999).

Figure 1 shows a schematic diagram of one version of the integrated model, the recently developed 12-Point Affect Circumplex (12-PAC; Yik et al., 2004). The space is arbitrarily carved into 12 segments (Segments I to XII), $30^{\circ}$ apart, on the metaphor of a clock. On the right hand side are the more pleasant states; on the left hand side the more unpleasant ones. On the upper half are the more activated states; on the lower half the more deactivated ones. Each segment thus represents a unique blend of pleasure

\footnotetext{
${ }^{2}$ Yik, Russell, and Steiger (2004) provided empirical estimates of the locations of Factors I and II within the IAS space. Factor I (extraversion) was about half way between PA and NO $\left(30^{\circ}\right.$ away from PA) and Factor II (agreeableness) about halfway between LM and JK (17 ${ }^{\circ}$ away from $\mathrm{LM}$ ). Together, this amounts to an average of $23.5^{\circ}$ rotation clockwise from Wiggins' axes of the IAS. The alternative rotation is indicated by dashed lines in Figure 2.
} 


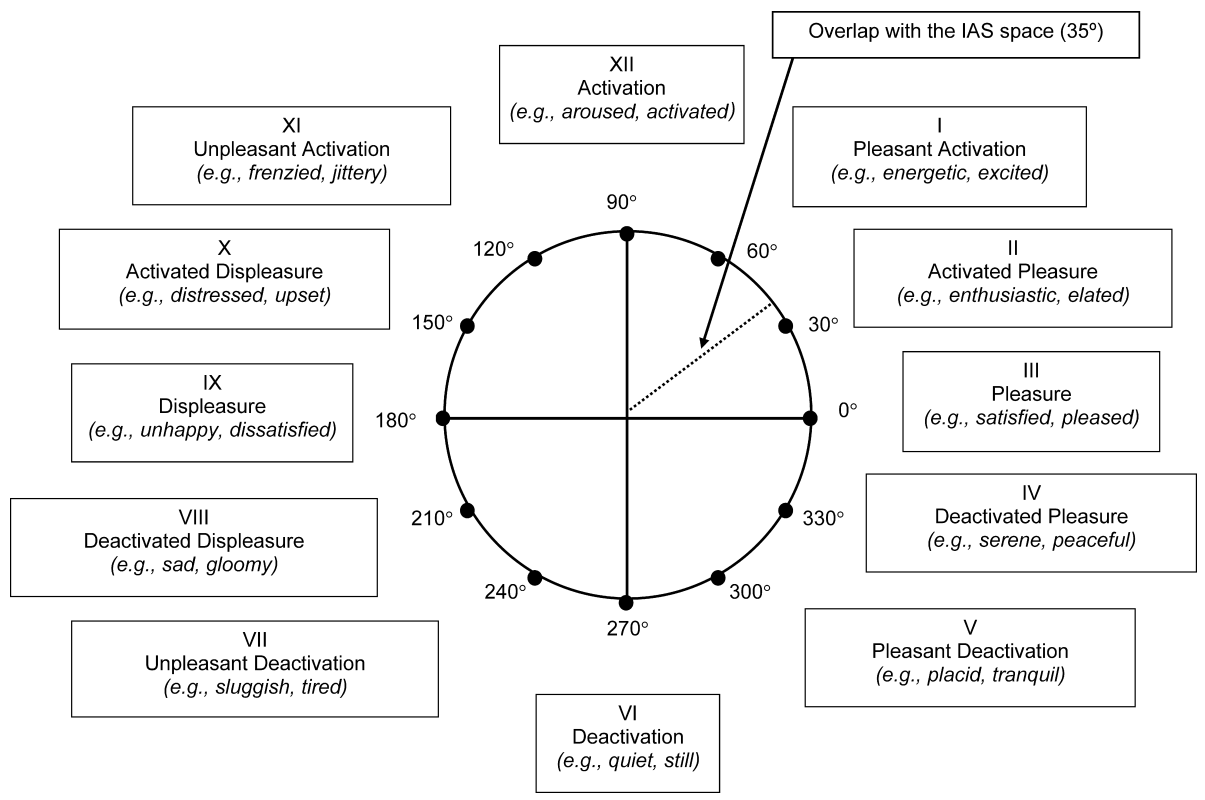

\section{Figure 1}

A 12-Point Affect Circumplex (12-PAC; Yik, Russell, \& Steiger, 2004). Figure shows a schematic diagram of the hypothetical locations of the 12 segments. Dashed line indicates the axis of overlap with the IAS circumplex.

and arousal. For instance, Segment I (characterized by a vector at $60^{\circ}$ ) captures states that are pleasant and very high in arousal, Segment II (a vector at $30^{\circ}$ ) captures those very pleasant and moderate in arousal. Actual affective states can fall anywhere on any vector throughout the space. The model is thoroughly bipolar in that any state has a bipolar opposite $180^{\circ}$ away. It is also a circumplex in which affective dimensions fall in a circular ordering along the perimeter rather than cluster at the axes. The circumplical nature of momentary affective states has received strong empirical support (Remington, Fabrigar, \& Visser, 2000; Yik et al., 2004).

\section{On the Relation between Affect and Personality}

At least since Galen proposed that temperament consists of emotions, investigators have speculated on close ties between personality and affect. Recently, Plutchik (1997) asserted that both phenomena come from the same 
biological source. Indeed, he speculated that many domains show a circumplex structure because they share the same biological source.

Affect and personality are known to be correlated (Carver, Sutton, \& Scheier, 2000; Fossum \& Feldman Barrett, 2000; Gross, Sutton, \& Ketelaar, 1998; Lucas \& Fijita, 2000; Moskowitz, Brown, \& Côté, 1997). The superfactors of Extraversion and Neuroticism, for example, yield robust relations to affect (Costa \& McCrae, 1980, 1984; Diener \& Emmons, 1984; Izard, Libero, Putnam, \& Haynes, 1993; McCrae \& Costa, 1991; Meyer \& Shack, 1989; O’Malley \& Gillett, 1984; Thayer, Takahashi, \& Pauli, 1988; Warr, Barter, \& Brownbridge, 1983; Watson \& Clark, 1992, 1997; Williams, 1981; Yik \& Russell, 2001; Yik, Russell, Ahn, Fernández Dols, \& Suzuki, 2002). The question that stimulated our study was how to describe the relation between a specific model of personality, Wiggins' (1995) IAS, and a specific model of affect, Yik et al.'s (2004) 12-PACS - both of which happen to be circumplexes.

A number of researchers have begun charting the relations between circumplexes. The most common approach has relied on a principal components analysis (PCA). With this approach, Alden et al. (1990) found the IAS and the Inventory of Interpersonal Problems scales shared a common two-factor space. Trobst (2000) placed the IAS scales within the space defined by her Support Actions Scales Circumplex scales (and vice versa) and concluded that the two measures overlapped on the horizontal axis (LM-DE). Saucier, Ostendorf, and Peabody (2001) found the IAS and their Non-Evaluative Personality Circumplex shared the vertical axis (PA$\mathrm{HI})$. To assess the validity of the interpersonal transaction cycle, Wagner, Kiesler, and Schmidt (1995) conducted a PCA on Impact Message Inventory (Kiesler, 1987) and Inventory of Interpersonal Problems (Alden et al., 1990) and found a structural convergence of these two circumplex measures on a common two-dimensional space. To demonstrate the convergent validity of his newly developed circumplex measure of interpersonal values, Locke (2000) conducted a PCA on the new values measures and the Inventory of Interpersonal Problems and again, found a structural convergence of the two measures. PCA has thus indicated empirical relations between circumplexes. However, as a very broad analytic tool, PCA is not ideally suited for detailing those relations. To the best of our knowledge, there is available no analytic technique built on the unique properties of the circumplex and thus tailored to chart the relationship between two circumplexes specifically.

Here we propose a new method to relate two circumplexes. We rely on CIRCUM-extension procedure (Michael Browne, personal communication, June 12, 1999) to place each circumplex within the other. Our 
method is described in detail in the Appendix. The remainder of this article reports the use of this method to describe the relationship between the IAS and the 12-PACS.

\section{Overview of the Current Study}

In the current study, a sample of 395 participants completed a battery of questionnaires that included Wiggins' (1995) IAS-R and Yiket al.'s (2004) 12-PACS. The affect data from the present study had been analyzed for the psychometric and structural properties of the affect scales and reported elsewhere (Yik et al., 2004). The affect scales were found to conform to a circumplex structure. Here we report additional analyses related to the IAS structure and its relation to affect. Specifically, we examine (a) how well the IAS variables conform to a circumplex; (b) the placement of the IAS variables within the 12-PACS space; (c) the placement of the 12-PACS variables within the IAS space; and (d) the location and nature of the overlap between the two circumplexes.

\section{Method}

\section{Procedure}

Participants were 395 undergraduates (144 men and 251 women) of a large Canadian university. They completed two affect questionnaires and then a personality questionnaire. On the first affect questionnaire, they were asked to describe affective feelings at a particular moment from either Yesterday or from Last Saturday. The specific moment during that day was selected with the help of one of six randomly assigned instructions (before breakfast, after breakfast, before lunch, after lunch, before dinner, and after dinner). The instructions then emphasized that all subsequent questionnaires were to be answered with respect to that selected moment. After the first affect questionnaire, the participants were given another, this time for a different day and a different time during the day. Half of the participants completed the Yesterday moment first and the remaining half completed the Last Saturday moment first.

\section{Affect Measures}

After selecting a specific moment, participants were asked to describe how they felt at that moment by completing the 12-PACS (Yik et al., 2004). The 12-PACS consists of three different response formats: (a) Adjective 
format, which is an adjective list accompanied by a five-point Likert scale ranging from 1 "not at all" to 5 "extremely"; (b) "Agree-Disagree" format, which is a list of statements with which participants are asked to indicate their degree of agreement, ranging from 1 "strongly disagree" to 5 "strongly agree"; and (c) "Describes Me" format, which is a list of statements, for each of which participants is asked to indicate how well it described their feelings, ranging from 1 "not at all" to 4 "very well". There are 75 items in the Adjective format; 61 in the "Agree-Disagree" format; and 55 in the "Describes Me" format.

The 12-PACS yield 36 affect scores (12 affect segments $\times 3$ response formats) for a given moment. To provide a preliminary estimate of a person's average affect, we summed the score for each affect segment from Yesterday with the corresponding score from Last Saturday. The typical correlation between corresponding scores, across the two moments, was modest. The mean correlation was $.07(S D=.07$; range: -.02 to .19$)$ for the 12 segments in the Adjective format; .10 for the "Agree-Disagree" format $(S D=.09$; range $=-.06$ to .21$)$; and .12 for the "Describes Me" format $(S D=.07$; range $=.00$ to .23$)$. These means justify combining the two moments, although the modest magnitude of the correlations suggest that a larger sample of moments would be better. For each affect segment, we transformed its three affect scales into their corresponding $z$-scores. We then computed 12 combined affect scores by summing across their respective $6 z$-scores $(2$ moments $\times 3$ scales). In the subsequent analyses, these combined scores were used.

\section{Personality Measures}

The last questionnaire began with the instruction "... describe yourself as you are GENERALLY and TYPICALLY." Participants completed Wiggins' (1995) 64-item Interpersonal Adjective Scales Revised (IAS-R) and other questionnaires used for other purposes.

IAS-R consists of eight octant scales each defined by eight adjectives: Assured-Dominance (PA), Arrogant-Calculating (BC), Cold-hearted (DE), Aloof-Introverted (FG), Unassured-Submissive (HI), Unassuming-Ingenuous (JK), Warm-Agreeable (LM), and Gregarious-Extraverted (NO). Participants were presented with a list of single adjectives and asked to rate the self-descriptive accuracy of each on an 8-point Likert scale ranging from "extremely inaccurate" to "extremely accurate." Each octant score was computed as an average of its eight constituent items. 


\section{Results \& Discussion}

Structure of the IAS

Preliminary tests. The pattern of correlations seen in the current data replicated previous findings (e.g., Wiggins, 1979) that had suggested a circumplex structure. For example, the correlations between a given octant variable and all other variables formed a cosine wave (Gurtman, 1991; Lorr \& McNair, 1963; Stern, 1970; Wiggins, 1979). Similarly, in an exploratory factor analysis, two principal components accounted for $78 \%$ of total variance. Inside the circle of Figure 2 are the coordinates of the eight octant variables on the first two unrotated principal components. The IAS scales show the predicted circular order. ${ }^{3}$

CIRCUM. With the encouraging results from these preliminary analyses, we used Browne's (1992) structural equation modeling program CIRCUM to provide a more rigorous test of circumplexity. This program used a maximum likelihood estimation technique to provide fit indices and an angular position for each input variable. LM was designated as the reference variable (its location was fixed at $0^{\circ}$ ), with the location of other variables then estimated relative to LM. The communality estimates of all variables were free to vary. No constraints were put on the minimum common score correlation (MCSC) nor on the estimates of the polar angles.

The analysis converged on a solution in 9 iterations. Three free parameters were specified in the correlation function equation; additional free parameters did not improve the model fit. The final model had a total of 26 free parameters and 10 degrees of freedom. The data fit the model well: ${ }^{4}$

\footnotetext{
${ }^{3}$ To examine the possibility of a general factor in the IAS data (Bentler, 1969), we extracted three principal components. The eigenvalues were 3.21, 3.00, and .60. The first two were bipolar factors as predicted by Wiggins. The third component was not a general factor, and indeed was difficult to interpret. We concluded that there was no evidence for a general factor in the IAS (see also Gurtman, 1994). Therefore, we decided not to ipsatize the data; instead, raw data were used in subsequent analyses.

${ }^{4}$ To assess the degree to which a circumplex model fits the observed data, we rely on two indices. First, the chi-square statistic $\left(\chi^{2}\right)$ was used. This statistic tests the null hypothesis that the hypothesized model reproduces the correlation matrix for the manifest variables. The larger the chi-square, the more the correlation matrix specified by the hypothesized model deviates from the correlation matrix for the manifest variables. The chi-square statistic is dependent on sample size, however, such that it can be significant even for models that fit the data relatively well (Bentler, 1990).

Second, Steiger and Lind's (1980) root mean square error of approximation (RMSEA), which can be regarded as a root mean square standardized residual, was used. RMSEA is adjusted for model complexity and is therefore useful in evaluating the degree of model fit. Greater values indicate poorer fit. A $90 \%$ confidence interval is given for each estimate.
} 


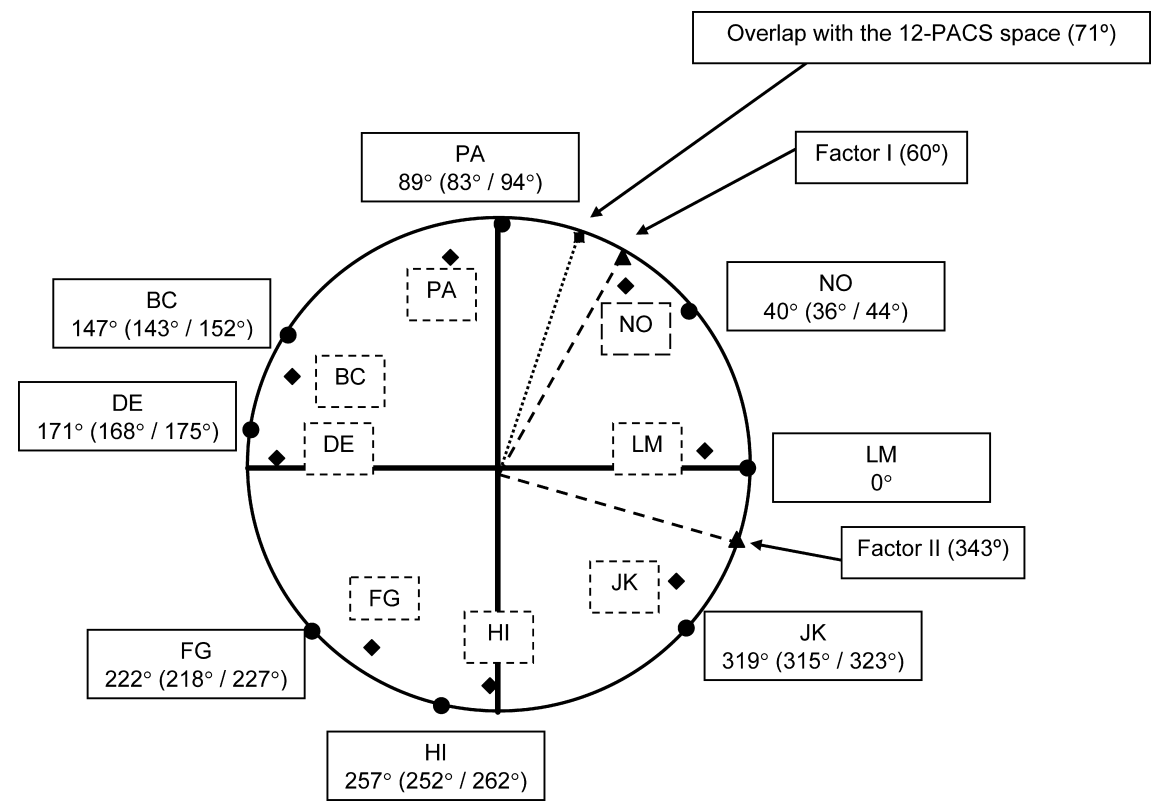

\section{Figure 2}

Wiggins' Interpersonal Adjective Scales (IAS) placed in a two-dimensional space via Principal Components Analysis (indicated by $\bullet$ on the inner part of the circle) and via CIRCUM (indicated by on the circumference of the circle). Figures given for CIRCUM are estimates of polar angles with $95 \%$ confidence intervals in parentheses. Dashed lines indicate possible alternate rotations: Factor I and II (empirical values from Yik, Russell, \& Steiger, 2004), and axis of overlap with the 12-PACS circumplex.

$\chi^{2}(10, N=395)=27.10$, RMSEA $=.07(90 \%$ confidence interval $=.04 / .10)$, MCSC $=-.85$. Similar results were obtained when communalities were constrained to be equal: $\chi^{2}(17, N=395)=62.97$, RMSEA $=.08(90 \%$ confidence interval $=.06 / .11), \mathrm{MCSC}=-.85$. For convenience, results from the model with constrained communality are shown on the circumference of the circle in Figure 2. CIRCUM placed the IAS variables reassuringly close to where our exploratory factor analysis and where previous tests for circumplexity had placed them (Gurtman \& Pincus, 2000).

\section{The Relationship Between the IAS and the 12-PACS}

Our focal question is how the IAS and the 12-PACS are related. We began by examining the zero-order correlations of each IAS variable with the 
12 affect variables. The circumplex provides a powerful prediction about the pattern of these correlations, namely, that they form a cosine wave (Yik \& Russell, 2001). Figure 3 shows a test of this prediction: the 12 affect variables form the abscissa; and the correlation of each with NO from the IAS is the ordinate. A similar plot was formed for each of the IAS variables. Some formed a cosine wave, but others were essentially flat. The maximal correlation (the peak of the cosine wave) and the location of that correlation also varied across the IAS variables. NO was chosen to illustrate the cosine wave in Figure 3 because it achieved the highest maximal correlation (.29) in this analysis. The variation in maximal correlation and location is made more precise in the next series of analyses.

In the Appendix, we propose a more general procedure for describing the relationship between two circumplexes, one of which is called the base, and the other called the secondary, circumplex. Our analysis was developed for a base circumplex completely accounted for by two dimensions and with the correlation between variables $180^{\circ}$ apart equal to -1.00 . Despite these constraints, as will be seen, the technique proved valuable. It is aimed at characterizing the relation between two circumplex structures as wholes. It estimates whether their relation involves zero, one, or both axes of the base circumplex; it estimates the degree of overlap on the involved axes; and it locates the vector of overlap within each circumplex.
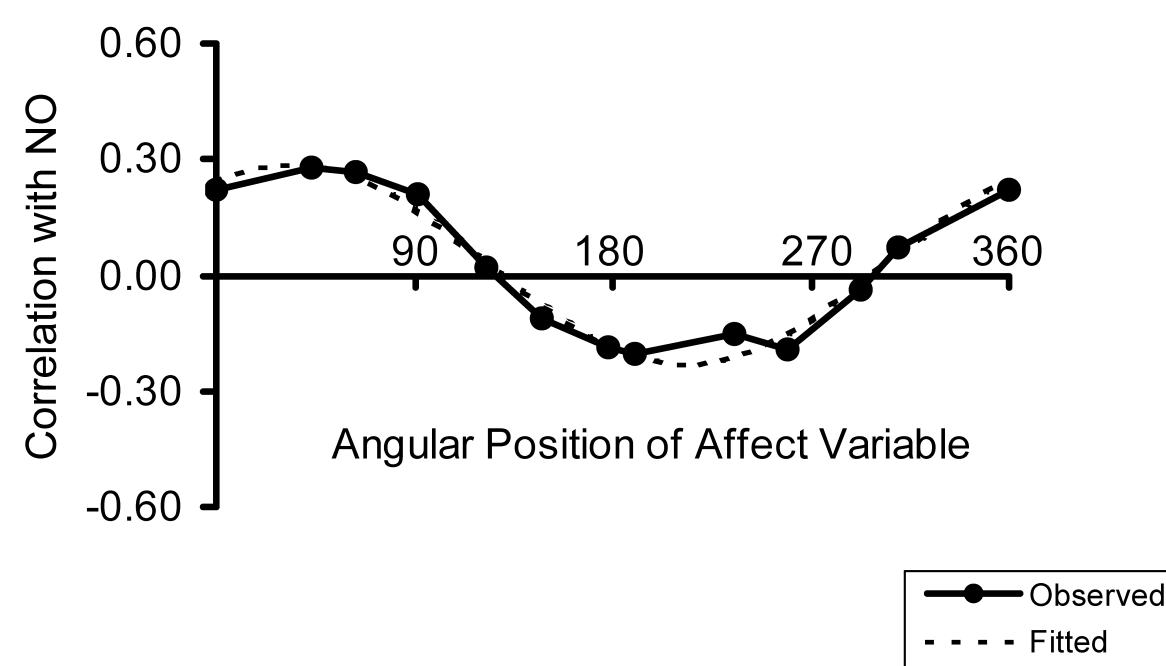

Figure 3

The correlation of NO (from the IAS) with each affect variable represented as its angle within the 12-PACS circumplex. The value for the affect variable at $0^{\circ}$ is repeated at $360^{\circ}$ to show the complete curve. 
We began with the 12-PAC as the base circumplex and then placed, by means of the CIRCUM-extension procedure (Michael Browne, personal communication, June 12, 1999), each IAS variable into the 12-PACS structure. Results are shown in Table 1. For each IAS variable, the first two figures simply indicate its place within the IAS structure: ${ }^{5} \zeta_{i}$ is a communality index (the square root of the proportion of variance of the variable that is explained by the CIRCUM model for the IAS structure); $\theta_{i}$ estimates the variable's location within the IAS space. The next two figures show each IAS variable's place within the 12-PACS structure: $\zeta_{+}$is a communality index (the square root of the proportion of variance of the IAS variable explained by the CIRCUM model for the 12-PACS structure); $\theta_{+}$is its estimated angle within the 12-PACS space.

Figure 4 is a plot of the $\zeta_{+}$for each IAS variable as a function of its location $\left(\theta_{i}\right)$ in the IAS space. A comparison of Figure 4 with the plots of simulated data in the Appendix suggests that the IAS is related to the 12PACS on one axis, although the degree of relationship is not large. That is,

Table 1

Empirical Locations of IAS Variables in the 12-PACS Space via CIRCUM$\underline{\text { extension }}$

\begin{tabular}{lccccc}
\hline & $\begin{array}{c}\text { Estimates within } \\
\text { the IAS Space }\end{array}$ & & \multicolumn{2}{c}{$\begin{array}{c}\text { Estimates when } \\
\text { placed within the } \\
\text { 12-PACS Space }\end{array}$} \\
\cline { 2 - 3 } \cline { 5 - 6 } IAS Variable & $\zeta_{i}$ & $\theta_{i}$ (degree) & & $\zeta_{+}$ & $\theta_{+}$(degree) \\
\hline Assured-Dominance (PA) & .96 & 89 & & .32 & 40 \\
Arrogant-Calculating (BC) & .96 & 146 & & .10 & 83 \\
Cold-hearted (DE) & .92 & 171 & & .05 & 195 \\
Aloof-Introverted (FG) & .93 & 222 & & .32 & 204 \\
Unassured-Submissive (HI) & .94 & 256 & & .34 & 217 \\
Unassuming-Ingenuous (JK) & .90 & 320 & & .01 & 256 \\
Warm-Agreeable (LM) & .91 & 0 & & .05 & 132 \\
Gregarious-Extraverted (NO) & 1.00 & 40 & & .34 & 36 \\
\hline
\end{tabular}

Note. $N=395$.

\footnotetext{
${ }^{5}$ Here we used the CIRCUM model with unconstrained communality because it achieved better overall fit.
} 
Figure 4 is unlike the results for either zero or two dimensions (Figure A1), but resembles those for one dimension of overlap (Figures A2 and A3). The pattern of two humps is unmistakable. There were two clusters of adjacent IAS variables with non-negligible values of $\zeta_{+}$(in the range .32 to .34 ), separated by negligible values (in the range .01 to .10). One cluster consists of $\mathrm{NO}$ and PA, the other of FG and HI.

Having concluded that one axis of the IAS overlaps the 12-PACS space, we turn to finding where. The procedure outlined in the Appendix allows us to estimate the angle within the 12-PACS (base) circumplex where the two circumplexes overlap. ${ }^{6}$ We examine those IAS variables with higher values of $\zeta_{+}$(in this case, $\geq .30$ ) in Table 1 . Variables NO and PA (with $\zeta_{+}$of .34 and .32) had $\theta_{+}$of $36^{\circ}$ and $40^{\circ}$. The mean of these numbers is $38^{\circ}$, and so we can estimate that the IAS space intersects the 12-PACS space at $38^{\circ}$. Variables FG and HI (with $\zeta_{+}$of .32 and .34), had $\theta_{+}$of $204^{\circ}$ and $217^{\circ}$. The mean of these numbers is $211^{\circ}$, and so we can estimate that the IAS overlaps the 12PACS at $211^{\circ}$. This result is comforting, because $38^{\circ}$ and $211^{\circ}$ differ by $173^{\circ}$, which is close to the anticipated $180^{\circ}$. For simplicity, we can assume that the axis of overlap is straight and passes through the center of the space (i.e., that the two points of overlap differ by $180^{\circ}$ ). This assumption suggests that the axis of overlap lies approximately at $35^{\circ}$ and $215^{\circ}$ in the 12 -PACS space. ${ }^{7}$

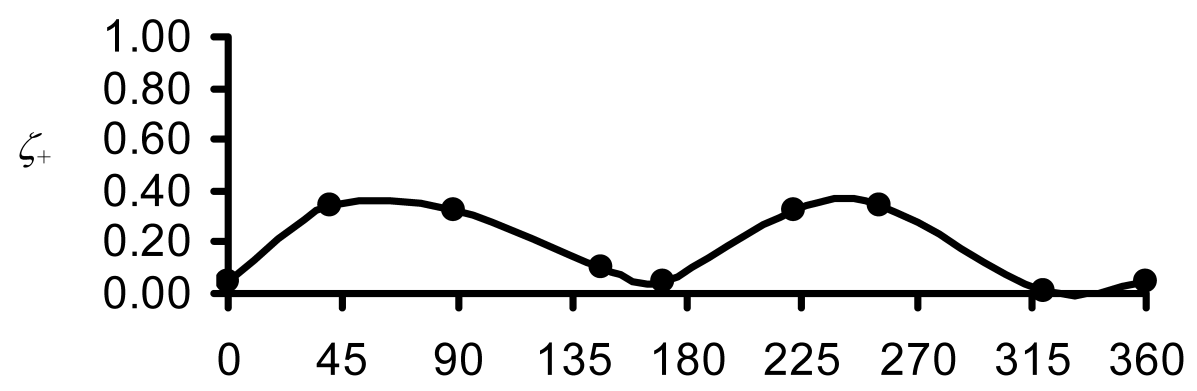

Location $\left(\theta_{i}\right)$ in the IAS space

\section{Figure 4}

Placing IAS variables into the 12-PACS space: $\zeta_{+}$of each IAS variable as a function of its location $\left(\theta_{i}\right)$ in the IAS space.

\footnotetext{
${ }^{6}$ We also repeated the analyses on two affect moments separately. Results were very similar to those obtained from the sum across two moments.

${ }^{7}$ Assuming that the axis of overlap is a straight line allows us to use both empirical values $\left(38^{\circ}\right.$ and $211^{\circ}$ ) to estimate the angular positions of that axis. The first estimate was $38^{\circ}$. The second was computed by subtracting $180^{\circ}$ from $211^{\circ}$, resulting in $31^{\circ}$. The average of $38^{\circ}$ and $31^{\circ}$ produced our final estimate of $35^{\circ}$. $215^{\circ}$ was simply the angle $180^{\circ}$ away.
} 
A separate question is where in the IAS space lies its axis of overlap with the 12-PACS space. We therefore carried out a parallel analysis with the IAS as base and 12-PACS as secondary. Results are illustrated in Figure 5 and given in Table 2. Again, two clusters surfaced. One cluster consists of Segments XII-I-II-III (with $\zeta_{+}$ranging from .25 to .32) with corresponding $\theta_{+}$ranging from $73^{\circ}$ to $96^{\circ}$. The mean of these estimates is $84^{\circ}$, and so we can estimate that the 12-PACS space overlaps the IAS space at $84^{\circ}$. The other cluster consisted of Segments VI-VII-VIII-IX (with $\zeta_{+}$ranging from .20 to .25 ) with corresponding $\theta_{+}$ranging from $229^{\circ}$ to $247^{\circ}$. The mean of these estimates is $238^{\circ}$, and so we can estimate that the 12-PACS overlaps the IAS at $238^{\circ}$ as well. The difference between $84^{\circ}$ and $238^{\circ}$ was $154^{\circ}$, a bit off from the anticipated $180^{\circ}$. Nevertheless, for simplicity, we can assume that the axis of overlap is straight (i.e., that the two points of overlap differ by $180^{\circ}$ ). This assumption (see footnote 6) suggests that the axis of overlap lies approximately at $71^{\circ}$ and $251^{\circ}$ in the IAS space.

\section{Conclusion}

Circumplex models have rightly enjoyed wide popularity in social and personality psychology. Among the applications in personality, Wiggins' (1995) IAS model elegantly exemplifies the usefulness of the circumplex in capturing in a small space the full spectrum of interpersonal dispositions. In relating Wiggins' circumplex to affect, our results showed that the nomological network of the IAS includes a connection to the 12-PACS, which had previously been shown to integrate major dimensional models of affect.

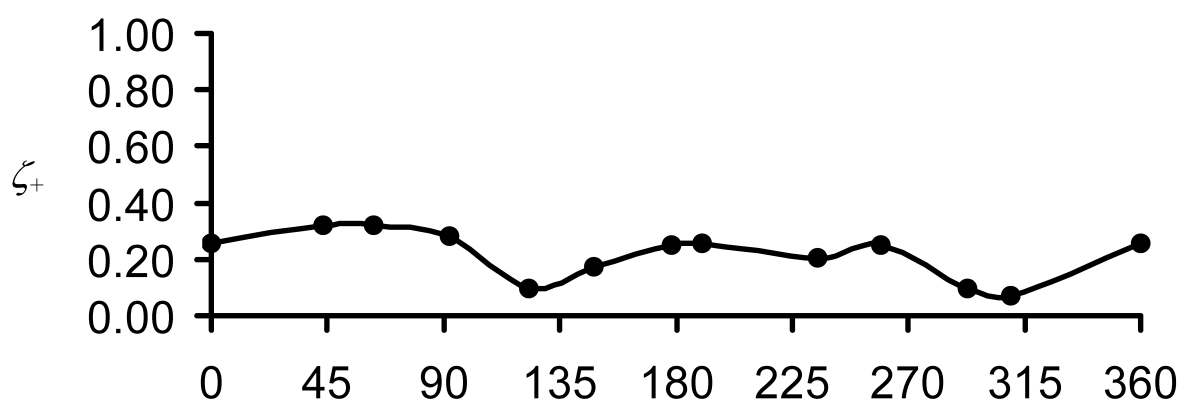

Location $\left(\theta_{i}\right)$ in the 12-PACS space

\section{Figure 5}

Placing the 12-PACS into the IAS space: $\zeta_{+}$of each 12-PACS variable as a function of its location $\left(\theta_{i}\right)$ in the 12-PACS space. 
Table 2

Empirical Locations of the 12-PACS Variables in the IAS Space via CIRCUMextension

$\begin{array}{cc}\text { Estimates within } & \text { Estimates when } \\ \text { the 12-PACS Space } & \text { placed within the } \\ & \text { IAS Space }\end{array}$

Affect

\begin{tabular}{llrrrr} 
Segment & Construct & $\zeta_{i}$ & $\theta_{i}$ (degree) & $\zeta_{+}$ & $\theta_{+}$(degree) \\
\hline I & Pleasant Activation & .97 & 63 & 0.32 & 84 \\
II & Activated Pleasure & .93 & 43 & 0.32 & 84 \\
III & Pleasure & .98 & 0 & 0.25 & 73 \\
IV & Deactivated Pleasure & .97 & 310 & 0.07 & 98 \\
V & Pleasant Deactivation & .99 & 293 & 0.09 & 200 \\
VI & Deactivation & .97 & 259 & 0.25 & 229 \\
VII & Unpleasant Deactivation & .93 & 235 & 0.20 & 235 \\
VIII & Deactivated Displeasure & .95 & 190 & 0.25 & 242 \\
IX & Displeasure & .98 & 178 & 0.25 & 247 \\
X & Activated Displeasure & .99 & 148 & 0.17 & 225 \\
XI & Unpleasant Activation & .96 & 123 & 0.10 & 151 \\
XII & Activation & .99 & 92 & 0.28 & 96 \\
\hline
\end{tabular}

Note. $N=395$.

How is one to interpret the interlocking relation between two circumplexes seen here? First, let us focus on the 12-PACS. Through the affective space runs an axis-a vector we estimate at $35^{\circ}$ in one direction and $215^{\circ}$ in the other. This axis contrasts two sets of opposite feelings. The vector at $35^{\circ}$ is characterized by pleasant affective feelings with moderately high arousal level; the vector at $215^{\circ}$ by unpleasant feelings with moderately low arousal level. It is along this axis that interpersonal dispositions are most strongly related to affect.

Next, focus on the IAS. Through this personality space runs an axis, a vector we estimate at $71^{\circ}$ in one direction and at $251^{\circ}$ in the other. The vector at $71^{\circ}$ lies between NO (Gregarious-Extraverted) and PA (AssuredDominance) and describes someone who is extraverted, outgoing, selfconfident, and self-assured. The vector at $251^{\circ}$ lies between FG (AloofIntroverted) and HI (Unassured-Submissive) and describes someone who is 
introverted, timid, bashful, and shy. It is along this axis that affect is most closely related to interpersonal dispositions. This axis is close to empirical estimates of where the Extraversion-Introversion factor of the Five Factor Model lies within the IAS. Our finding is thus uncannily consonant with McCrae and Costa's (1989) prescient argument that affect and interpersonal dispositions are related because "... affects and interpersonal behaviors have a common cause: the underlying dimension of Extraversion. Structurally, one could say that the dimension of Extraversion is defined by the intersection of the affective plane with the interpersonal plane" (p. 593).

These results are summarized graphically in Figure 6, which provides two different views of the overlap of those two planes. Because structural models can be rotated with no loss of fit to the data, the question of the best rotation is sometimes discussed in terms of relations to external variables (Larsen \& Diener, 1992). If axes are rotated to the place where the structure maximally relates to external variables, then our findings show that the 12-PACS intersects the IAS (at $\left.71^{\circ}\right)$ close to where Extraversion $\left(60^{\circ}\right.$ in Figure 2) overlaps the IAS. Parallel considerations apply to the rotation of the axes within the 12-PACS. The entire IAS space overlaps the 12-PACS space on the pleasant activated versus unpleasant deactivated axis. This axis corresponds to neither Pleasure nor Arousal as envisioned by Russell (1980), but does approximately correspond to the Positive Activation dimension envisioned by Watson and Tellegen (1985).

The magnitude of relation found between the IAS and the 12-PACS was modest. Indeed, a not unreasonable conclusion is that the values of $\zeta_{+}$ obtained here are far from the values expected when two circumplexes share one or both axes. Nevertheless, at least two considerations suggest that we are underestimating that relation. First, personality is related to affect not so much at a given moment (with the idiosyncrasies of time and place), but in the aggregate (Epstein, 1979). Here we averaged two moments of affective experience, but two is a rather small sample size. Analyses (mentioned in Footnote 4) showed that the form of the relation between IAS and 12-PACS did not differ even when only one moment was analyzed; nevertheless, the magnitude of the relation would surely rise as the sample of moments increases in size. Second, our sample of moments was quasi-random. Our procedure captured moments when the participant was alone as well as moments with others. Interpersonal dispositions summarized in Wiggins' IAS can be expected to predict affective reactions during interpersonal encounters, but are silent about a person's affect when alone. Indeed, even a sample of interpersonal moments would be but a first approximation, because there are different types of situations differentially interacting with specific dispositions. An important question for future 
M. Yik and J. Russell
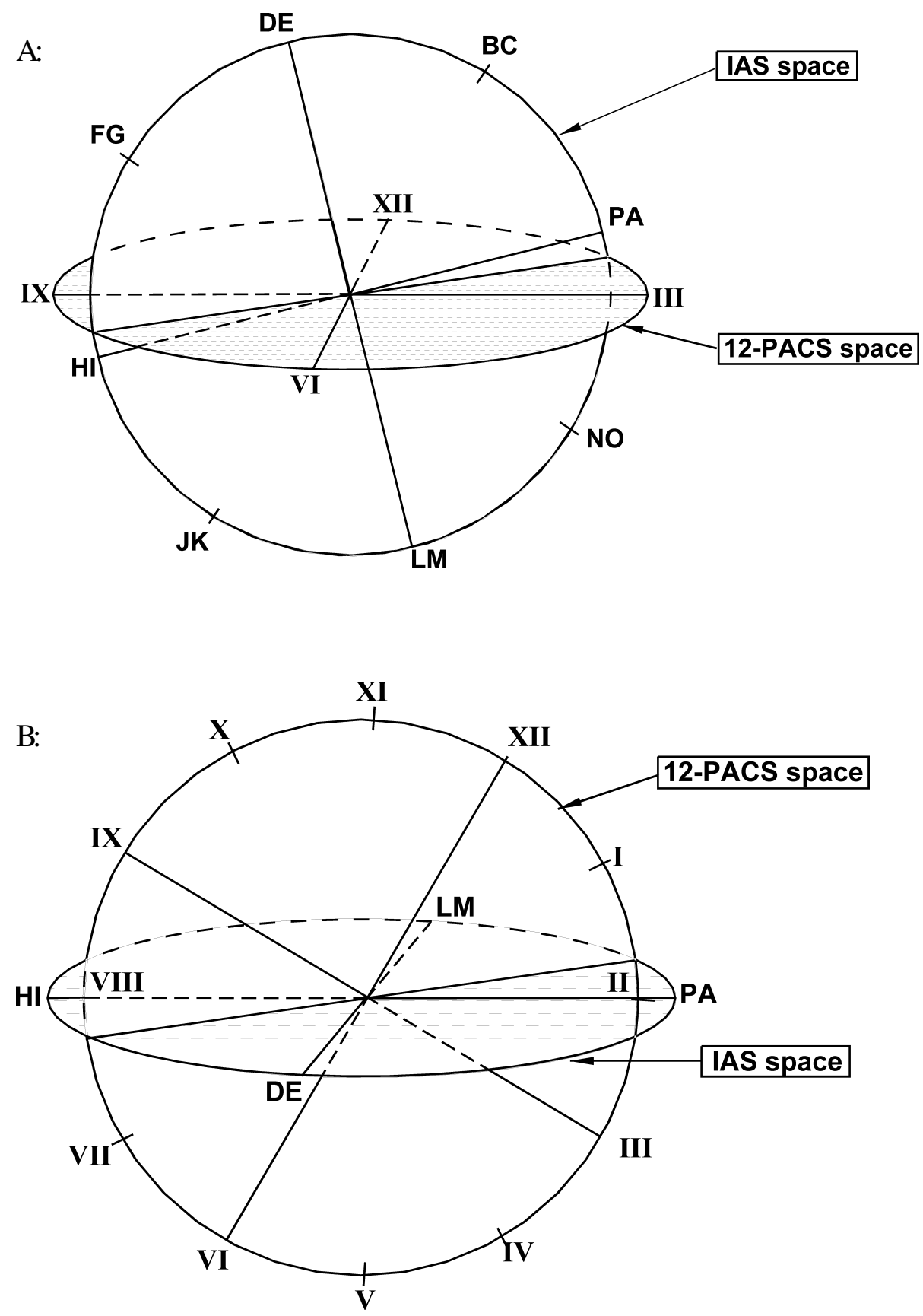

\section{Figure 6}

A: Overlap of the IAS Circumplex with the 12-PACS Circumplex

B: Overlap of the 12-PACS Circumplex with the IAS Circumplex 
research is therefore whether the relation between the IAS and 12-PACS found here remains the same when affect is sampled in specific categories of interpersonal situations.

Admittedly, our proposed method of relating two circumplex models is preliminary. Further efforts should be directed at creating a more mathematically sophisticated and more general procedure applicable to circumplexes of any type. By showing that empirical data are illuminated by the results obtained with our simulations, the present results show that this development would be a worthwhile enterprise.

\section{References}

Alden, L. E., Wiggins, J. S., \& Pincus, A. L. (1990). Construction of circumplex scales for the Inventory of Interpersonal Problems. Journal of Personality Assessment, 55, 521-536.

Almagor, M. \& BenPorath, Y. (1989). The two-factor model of self-reported mood: A crosscultural replication. Journal of Personality Assessment, 53, 10-21.

Benjamin, L. S. (1974). Structural analysis of social behavior. Psychological Review, 81, 392-425.

Bentler, P. M. (1969). Semantic space is (approximately) bipolar. Journal of Psychology, 71, 33-40.

Bentler, P. M. (1990). Comparative fit indexes in structural models. Psychological Bulletin, 107, 238-246.

Browne, M. W. (1992). Circumplex models for correlation matrices. Psychometrika, 57, 469-497.

Buss, D. M. (1989). Conflict between the sexes: Strategic interference and evocation of anger and upset. Journal of Personsality and Social Psychology, 56, 735-747.

Buss, D. M. \& Barnes, M. (1986). Preferences in human mate selection. Journal of Personality and Social Psychology, 50, 559-570.

Carver, C. S., Sutton, S. K., \& Scheier, M. F. (2000). Action, emotion, and personality: Emerging conceptual integration. Personality and Social Psychology Bulletin, 26, 741-751.

Church, A. T., Katigbak, M. S., \& Reyes, J. A. S., \& Jensen, S. M. (1999). The structure of affect in a non-Western culture: Evidence for cross-cultural comparability. Journal of Personality, 67, 503-532.

Costa, P. T., Jr. \& McCrae, R. R. (1980). Influence of extraversion and neuroticism on subjective well-being: Happy and unhappy people. Journal of Personality and Social Psychology, 38, 668-678.

Costa, P. T., Jr. \& McCrae, R. R. (1984). Personality as a lifelong determinant of wellbeing. In C. Z. Malatesta \& C. E. Izard (Eds.), Emotion in adult development (pp. 141-157). Beverly Hills: Sage.

Diener, E. \& Emmons, R. A. (1984). The independence of positive and negative affect. Journal of Personality and Social Psychology, 47, 1105-1117.

Epstein, S. (1979). The stability of behavior: 1. On predicting most of the people much of the time. Journal of personality and Social Psychology, 37, 1097-1126.

Fabrigar, L. R., Visser, P. S., \& Browne, M. W. (1997). Conceptual and methodological issues in testing the circumplex structure of data in personality and social psychology. Personality and Social Psychology Review, 1, 184-203.

Foa, U. G. \& Foa, E. B. (1974). Societal structures of the mind. Springfield, IL: Charles C. Thomas. 


\section{Yik and J. Russell}

Fossum, T. A. \& Feldman Barrett, L. F. (2000). Distinguishing evaluation from description in the personality-emotion relationship. Personality and Social Psychology Bulletin, 26, 669-678.

Gaines, S. O., Jr., Panter, A. T., Lyde, M. D., Steers, W. N., Rusbult, C. E., Cox, C. L., \& Wexler, M. O. (1997). Evaluative the circumplexity of interpersonal traits and the manifestation of interpersonal traits in interpersonal trust. Journal of Personality and Social Psychology, 73, 610-623.

Gifford, R. (1991). Mapping nonverbal behavior on the Interpersonal Circle. Journal of Personality and Social Psychology, 61, 279-288.

Goldberg, L. R. (1977, August). Language and personality: Developing a taxonomy of traitdescriptive terms. Paper presented at the Division of Evaluation and Measurement at the annual meeting of the American Psychological Association, San Francisco.

Gross, J. J., Sutton, S. K., \& Ketelaar, T. (1998). Relations between affect and personality: Support for the affect-level and affective-reactivity views. Personality and Social Psychology Bulletin, 24, 279-288.

Gurtman, M. B. (1991). Evaluating the interpersonalness of personality scales. Personality and Social Psychology Bulletin, 17, 670-677.

Gurtman, M. B. (1994). The circumplex as a tool for studying normal and abnormal personality: A methodological primer. In S. Strack \& M. Lorr (Eds.), Differentiating normal and abnormal personality (pp. 243-263). New York: Springer.

Gurtman, M. B. \& Pincus, A. L. (2000). Interpersonal Adjective Scales: Confirmation of circumplex structure from multiple perspectives. Personality and Social Psychology Bulletin, 26, 374-384.

Guttman, L. (1954). A new approach to factor analysis: The radex. In P. R. Lazarsfeld (Ed.), Mathematical thinking in the social sciences (pp. 258-348). Glencoe, IL: Free Press.

Holland, J. L. (1973). Making vocational choices: A theory of careers. Englewood Cliffs, NJ: Prentice Hall.

Izard, C. E., Libero, D. Z., Putname, P., \& Haynes, O. M. (1993). Stability of emotion experiences and their relations to traits of personality. Journal of Personality and Social Psychology, 64, 847-860.

Joiner, T. E., Jr., Sandín, B., Chorot, P., Lostao, L., \& Marquina, G. (1997). Development and factor analytic validation of the SPANAS among women in Spain: (More) cross-cultural convergence in the structure of mood. Journal of Personality Assessment, 68, 600-615.

Kiesler, D. J. (1983). The 1982 Interpersonal Circle: A taxonomy for complementarity in human transactions. Psychological Review, 90, 185-214.

Kiesler, D. J. (1987). Research manual for the Impact Message Inventory. Palo Alto, CA: Consulting Psychologists Press.

Kiesler, D. J. (1996). Contemporary interpersonal theory and research. New York: John Wiley.

Larsen, R. J. \& Diener, E. (1992). Promises and problems with the circumplex model of emotion. In M. S. Clark (Ed.), Review of Personality and Social Psychology: Emotion (Volume 13, pp. 25-59). Newbury Park, CA: Sage.

Leary, T. (1957). Interpersonal diagnosis of personality. New York: Ronald Press.

Locke, K. D. (2000). Circumplex scales of interpersonal values: Reliability, validity, and applicability to interpersonal problems and personality disorders. Journal of Personality Assessment, 75, 249-267.

Lorr, M. \& McNair, D. M. (1963). An interpersonal behavior circle. Journal of Abnormal and Social Psychology, 67, 68-75.

Lorr, M. \& McNair, D. M. (1965). Expansion of the interpersonal behavior circle. Journal of personality and Social Psychology, 2, 823-830. 
Lucas, R. E. \& Fujita, F. (2000). Factors influencing the relation between extraversion and pleasant affect. Journal of Personality and Social Psychology, 79, 1039-1056.

McCrae, R. R. \& Costa, P. T., Jr. (1989). The structure of interpersonal traits: Wiggins circumplex and the five-factor model. Journal of Personality and Social Psychology, 56, 586-595.

McCrae, R. R. \& Costa, P. T., Jr. (1991). Adding Liebe und Arbeit: The full five-factor model and well-being. Personality and Social Psychology Bulletin, 17, 227-232.

Meyer, G. J. \& Shack, J. R. (1989). Structural convergence of mood and personality: Evidence for old and new directions. Journal of Personality and Social Psychology, 57, 691-706.

Moskowitz, D. S., Brown, K. W., \& Côté, S. (1997). Reconceptualizing stability: Using time as a psychological dimension. Current Directions in Psychology Science, 6, 127-132.

O’Malley, M. N. \& Gillette, C. S. (1984). Exploring the relations between traits and emotions. Journal of Personality, 52, 274-284.

Pincus, A. L. (2002). Constellations of dependency within the five-factor model of personality. In P. T. Costa, Jr. \& T. A. Widiger (Eds.), Personality disorders and the five-factor model of personality (2nd ed., pp. 203-214). Washington, DC: American Psychological Association.

Plutchik, R. (1997). The circumplex as a general model of the structure of emotions and personality. In R. Plutchik \& H. R. Conte (Eds.), Circumplex models of personality and emotions (pp. 17-45). Washington, DC: American Psychological Association.

Plutchik, R. \& Conte, H. R. (Eds.). (1997). Circumplex models of personality and emotions. Washington, DC: American Psychological Association.

Remington, N. A., Fabrigar, L. R., \& Visser, P. S. (2000). Re-examining the circumplex model of affect. Journal of Personality and Social Psychology, 79, 286-300.

Russell, J. A. (1980). A circumplex model of affect. Journal of Personality and Social Psychology, 39, 1161-1178.

Russell, J. A. (1983). Pancultural aspects of the human conceptual organization of emotions. Journal of Personality and Social Psychology, 45, 1281-1288.

Russell, J. A., Lewicka, M., \& Niit, T. (1989). A cross-cultural study of a circumplex model of affect. Journal of Personality and Social Psychology, 57, 848-856.

Saucier, G., Ostendorf, F., \& Peabody, D. (2001). The non-evaluative circumplex of personality adjectives. Journal of Personality, 69, 537-582.

Schaefer, E. S. (1959). A circumplex model for maternal behavior. Journal of Abnormal and Social Psychology, 59, 226-235.

Shepard, R. N. (1962). Analysis of proximities: Multidimensional scaling with an unknown distance function II. Psychometrika, 27, 219-246.

Steiger, J. H. \& Lind, J. C. (1980, May). Statistically-based tests for the number of common factors. Paper presented at the annual Spring Meeting of the Psychometric Society in Iowa City, IA.

Stern, G. G. (1970). People in context: Measuring person-environment congruence in education and industry. New York: Wiley.

Sullivan, H. S. (1953). The interpersonal theory of psychiatry. New York: Norton.

Thayer, R. E. (1996). The origin of everyday moods: Managing energy, tension, and stress. New York: Oxford University Press.

Thayer, R. E., Takahashi, P. J., \& Pauli, J. A. (1988). Multidimensional arousal states, diurnal rhythms, cognitive and social processes, and extraversion. Personality and Individual Differences, 9, 15-24.

Tracey, T. J. G. (2000). Analysis of circumplex models. In H. E. A. Tinsley \& S. D. Brown (Eds.), Handbook of applied multivariate statistics and mathematical modeling (pp. 641664). San Diego: Academic Press. 


\section{Yik and J. Russell}

Tracey, T. J. G. \& Schneider, P. L. (1995). An evaluation of the circular structure of the Checklist of Interpersonal Transactions and the Checklist of Psychotherapy Transactions. Journal of Counseling Psychology, 42, 496-507.

Trapnell, P. D. \& Wiggins, J. S. (1990). Extension of the Interpersonal Adjective Scales to include the big five dimensions of personality. Journal of personality and Social Psychology, 59, 781-790.

Trobst, K. K. (2000). An interpersonal conceptualization and quantification of social support transactions. Personality and Social Psychology Bulletin, 26, 971-986.

Wagner, C. C., Kiesler, D. J., \& Schmidt, J. A. (1995). Assessing the interpersonal transaction cycle: Convergence of action and reaction interpersonal circumplex measures. Journal of Personality and Social Psychology, 69, 938-949.

Warr, P., Barter, J., \& Brownbridge, G. (1983). On the independence of positive and negative affect. Journal of Personality and Social Psychology, 44, 644-651.

Watson, D. \& Clark, L. A. (1992). On traits and temperament: General and specific factors of emotional experience and their relation to the Five-Factor Model. Journal of Personality, 60, 441-476.

Watson, D. \& Clark, L. A. (1997). Extraversion and its positive emotional core. In R. Hogan, J. Johnson, \& S. Briggs (Eds.), Handbook of personality psychology (pp. 767-793). San Diego: Academic Press.

Watson, D., Clark, L. A., \& Tellegen, A. (1984). Cross-cultural convergence in the structure of mood: A Japanese replication and a comparison with U.S. findings. Journal of Personality and Social Psychology, 47, 127-144.

Watson, D. \& Tellegen, A. (1985). Toward a consensual structure of mood. Psychological Bulletin, 98, 219-235.

Widiger, T. A., Trull, T. J., Clarkin, J. F., Sanderson, C. J., \& Costa, P. T., (1994). A description of the DSM-III-R and DSM-IV personality disorders with the Five-Factor Model of personality. In P. T. Costa \& T. A. Widiger (Eds.), Personality disorders and the FiveFactor Model of personality (pp. 41-56). Washington, DC: American Psychological Association.

Wiggins, J. S. (1979). A psychological taxonomy of trait-descriptive terms: The interpersonal domain. Journal of Personality and Social Psychology, 37, 395-412.

Wiggins, J. S. (1995). Interpersonal Adjective Scales: Professional manual. Odessa, FL: Psychological Assessment Resources.

Wiggins, J. S. \& Pincus, A. L. (1989). Conceptions of personality disorders and dimensions of personality. Psychological Assessment, 1, 305-316.

Williams, D. G. (1981). Personality and mood: State-trait relationships. Personality and Individual Differences, 2, 303-309.

Yik, M. S. M. \& Russell, J. A. (2001). Predicting the big two of affect from the big five of personality. Journal of Research in Personality, 35, 247-277.

Yik, M. S. M. \& Russell, J. A. (2003). Chinese Affect Circumplex: I. Structure of recalled momentary affect. Asian Journal of Social Psychology, 6, 185-200.

Yik, M. S. M., Russell, J. A., Ahn, C.-K. (2003). Affect among Koreans: New scales and their structure. Korean Journal of Psychology, 22, 115-136.

Yik, M. S. M., Russell, J. A., Ahn, C.-K., Fernández Dols, J. M., \& Suzuki, N. (2002). Relating the Five-Factor Model of personality to a circumplex model of affect: A five language study. In R. R. McCrae \& J. Allik (Eds.), The Five-Factor Model of personality across cultures (pp. 79-104). New York: Kluwer Academic/Plenum Publishers.

Yik, M. S. M., Russell, J. A., \& Feldman Barrett, L. (1999). Structure of self-reported current affect: Integration and beyond. Journal of Personality and Social Psychology, 77, 600-619. 
Yik, M. S. M., Russell, J. A., Oceja, L. V., \& Fernández Dols, J. M. (2000). Momentary affect in Spanish: Scales, structure, and relation to personality. European Journal of Psychological Assessment, 16, 160-176.

Yik, M. S. M., Russell, J. A., \& Steiger, J. H. (2004). A 12-point circumplex model of core affect. Manuscript submitted for publication.

Yik, M. S. M., Russell, J. A., \& Suzuki, N. (2003). Relating momentary affect to the Five Factor Model of personality: A Japanese case. Japanese Psychological Research, 45, 80-93.

Appendix

\section{Describing the Relationship between Two Circumplexes}

To our knowledge, no comprehensive method for describing the relationship between two circumplexes is available. We therefore pressed CIRCUM (Browne, 1992) and CIRCUM-extension (Michael Browne, personal communication, June 12, 1999) into service for this purpose.

We begin with a set of variables that are known to form a circumplex, which we call the base circumplex. This set of variables is first analyzed with CIRCUM. The CIRCUM model associates two parameters with each variable: a polar angle, $\theta_{i}$, which locates the $i^{\text {th }}$ variable on the circumference of a circle, and a communality index, $\zeta_{i}$, which is the square root of the proportion of variance of that variable that is explained by the model. The CIRCUM model also provides the correlation function that fits the variables. The CIRCUM-extension procedure is then used to locate an outside variable on the circumference of the base circumplex by means of an estimated polar angle $\theta_{+}$and to determine the amount of variance of the outside variable explained by the base circumplex by means of an estimated communality index, $\zeta_{+}$. The procedure is accomplished by fitting a cosine-based correlation function to a vector of correlations between the outside variable and the variables constituting the base circumplex. A $\zeta_{+}$approaching zero indicates that the outside variable bears no relation with all of the variables of the base circle; consequently, the estimated polar angle $\theta_{+}$would not locate it precisely on the circumference of the base circumplex. A reasonable size of $\zeta_{+}$indicates that the position of the outside variable is located precisely on the circumference of the base circumplex. In the procedures to be described here, we used CIRCUM-extension to place each variable one at a time from a secondary circumplex onto the base circumplex. (Obviously, what is base and what is secondary can be reversed.)

Our treatment is informal and preliminary. We conducted a series of simulations with one base circumplex and different secondary circumplexes, each of known properties and with a known relationship to the base. For convenience, we created the base circumplex with 12 evenly spaced 
variables $\left(30^{\circ}\right.$ apart $)$, and each secondary circumplex with 8 evenly spaced variables $\left(45^{\circ}\right.$ apart). The base circumplex was a special case of a circumplex in which true scores on all variables are accounted for by two dimensions, in which variables are evenly spaced, and in which the variables at $180^{\circ}$ correlate -1.00 in the population (i.e., $\rho_{180}=-1.00$ ). In each simulation, we sampled data from both the base and the secondary circumplexes, calculated the resulting $20 \times 20$ correlation matrix, applied CIRCUM to each, then CIRCUM-extension to each variable of the secondary circumplex, and finally examined the resulting $\theta_{+}$and $\zeta_{+}$for each of the 8 variables in the secondary circumplex. In all cases, CIRCUM showed that the base and secondary structures created fit the circumplex model well.

Because the base circumplex is fully accounted for by two dimensions, there were only three possible types of linear relation between the base and the secondary: the secondary circumplex is correlated with the base circumplex along no dimension (i.e., is independent of the base circumplex), along one dimension, or along both. We therefore examine three cases: the secondary circumplex is linearly related to both, one, or neither of the axes of the base circumplex. In the first two cases, we begin by examining data in which the degree of relation is maximal and then turn to smaller degrees of relation. In all, we relied on 4 random variates $(x, y, u, v)$ which were standard normal (mean of 0 and a $S D$ of 1.00). We also created "random error" variables, by which we mean random normal variates with a mean of 0 and a $S D$ of 0.10 . For all 11 simulated circumplexes, we relied on the general formula for a vector $\mathbf{z}$ at angle $a^{\circ}$ :

$$
\mathbf{z}=\mathbf{x} * \cos \left(a^{\circ}\right)+\mathbf{y} * \sin \left(a^{\circ}\right)
$$

where $\mathbf{x}$ is the vector defining the horizontal axis and $\mathbf{y}$ the vector defining the vertical axis of the simulated circumplex.

Base Circumplex. To create the base circumplex $\left(C_{0}\right)$, we began with two of the random variates, $x$ and $y$. We then created 12 variables, each a linear combination of $x$ and $y$. That is, we used Equation 1 to create 12 variables as follows: $x * \cos (0)+y * \sin (0) ; x * \cos \left(30^{\circ}\right)+y * \sin \left(30^{\circ}\right) ; x *$ $\cos \left(60^{\circ}\right)+y * \sin \left(60^{\circ}\right) ; x * \cos \left(90^{\circ}\right)+y * \sin \left(90^{\circ}\right) ; x * \cos \left(120^{\circ}\right)+y *$ $\sin \left(120^{\circ}\right) ; x * \cos \left(150^{\circ}\right)+y * \sin \left(150^{\circ}\right) ; x * \cos \left(180^{\circ}\right)+y^{*} \sin \left(180^{\circ}\right) ; x *$ $\cos \left(210^{\circ}\right)+y * \sin \left(210^{\circ}\right) ; x * \cos \left(240^{\circ}\right)+y * \sin \left(240^{\circ}\right) ; x * \cos \left(270^{\circ}\right)+y *$ $\sin \left(270^{\circ}\right) ; x * \cos \left(300^{\circ}\right)+y * \sin \left(300^{\circ}\right) ; x * \cos \left(330^{\circ}\right)+y * \sin \left(330^{\circ}\right)$. To each of these 12 , we added a separate "random error" variable. 
Two Independent Circumplexes. To create a secondary circumplex $\left(C_{1}\right)$ completely independent of the base, $C_{1}$ was formed from two different random variates, $u$ and $v$, which were independent of the variates ( $x$ and $y$ ) used to form the base circumplex. In other words, using Equation 1, we created 8 variables, each a linear combination of $u$ and $v: u^{*} \cos \left(0^{\circ}\right)+v *$ $\sin \left(0^{\circ}\right) ; u^{*} \cos \left(45^{\circ}\right)+v^{*} \sin \left(45^{\circ}\right) ; u^{*} \cos \left(90^{\circ}\right)+v^{*} \sin \left(90^{\circ}\right) ; u^{*} \cos \left(135^{\circ}\right)$ $+v^{*} \sin \left(135^{\circ}\right) ; u^{*} \cos \left(180^{\circ}\right)+v^{*} \sin \left(180^{\circ}\right) ; u^{*} \cos \left(225^{\circ}\right)+v^{*} \sin \left(225^{\circ}\right)$, $u^{*} \cos \left(270^{\circ}\right)+v^{*} \sin \left(270^{\circ}\right) ; u^{*} \cos \left(315^{\circ}\right)+v^{*} \sin \left(315^{\circ}\right)$. To each of these 8 , we added a separate "random error" variable.

The base circumplex $C_{0}$ and the secondary circumplex $C_{1}$ formed in this way had a total of 20 variables. For these 20 , we sampled 1000 cases. We then located each of the 8 variables from the secondary circumplex on the circumference of the base circumplex, one at a time, through CIRCUMextension. The bottom-most line (composed of $\mathbf{\square}$ ) of Figure A1 shows the $\zeta_{+}$for each of the 8 , plotted as a function of the variable's angle $\left(\theta_{i}\right)$ within the secondary circumplex. Values of $\zeta_{+}$ranged from .01 to .06 . Because $\zeta_{+}$ cannot take on negative values, it is a biased estimator of the true relationship, which has been set to zero in this case. When base and secondary circumplex bear no relationship, CIRCUM-extension can fit error variance to arrive at a small but positive $\zeta_{+}$. Nevertheless, all $\zeta_{+}$values we obtained were small enough to suggest no relation. Because the $\zeta_{+}$were negligible, the $\theta_{+}$were not meaningful. In summary, the results show that application of CIRCUM-extension can reveal when two circumplexes are unrelated. Small values of $\zeta_{+}$(up to .06 with an $N$ of 1000 but presumably higher with smaller $N$ ) indicate unrelated circumplexes.

Overlap on Both Axes of the Base Circumplex. We next examine the opposite extreme: the case of two identical circumplexes. That is, the secondary circumplex $\left(C_{2}\right)$ was formed from the same two random variates used to form the base circumplex, $x$ and $y$. Relying on Equation 1, we created 8 variables, each a linear combination of $x$ and $\mathrm{y}: x * \cos \left(0^{\circ}\right)+y *$ $\sin \left(0^{\circ}\right) ; x * \cos \left(45^{\circ}\right)+y * \sin \left(45^{\circ}\right) ; x * \cos \left(90^{\circ}\right)+y * \sin \left(90^{\circ}\right) ; x *$ $\cos \left(135^{\circ}\right)+y * \sin \left(135^{\circ}\right) ; x * \cos \left(180^{\circ}\right)+y * \sin \left(180^{\circ}\right) ; x * \cos \left(225^{\circ}\right)+y *$ $\sin \left(225^{\circ}\right), x * \cos \left(270^{\circ}\right)+y^{*} \sin \left(270^{\circ}\right) ; x * \cos \left(315^{\circ}\right)+y * \sin \left(315^{\circ}\right)$. To each of these 8 , we added a separate "random error" variable.

The base circumplex and the secondary circumplex formed in this way had a total of 20 variables. For these, we sampled 1000 cases. We then used CIRCUM-extension to add to the base circumplex each of the 8 variables of the secondary circumplex, one at a time. Figure A1 (the topmost line composed of $\diamond$ ) shows the $\zeta_{+}$for each of the 8 , plotted as a function of the variable's angle $\left(\theta_{i}\right)$ within the secondary circumplex. Values of $\zeta_{+}$were 
all 1.00, clearly revealing the relation between the two circumplexes. These results show that application of CIRCUM-extension can reveal when two circumplexes are fully overlapping on both axes. In this case, values of $\theta_{+}$ reveal the angle within the base for each variable from the secondary circumplex.

We next turn to secondary circumplexes bearing smaller (but equal) degrees of overlap with both axes of the base. To create circumplexes with different degrees of overlap with the axes of the base circumplex, we relied on a procedure provided by Michael Browne (personal communication, September 4, 2003): Let $p_{x}$ represent the proportion of variance of the horizontal axis of the secondary circumplex explained by the base circumplex. Let $p_{y}$ represent the proportion of variance of the vertical axis of the secondary circumplex explained by the base circumplex. Both $p_{x}$ and $p_{y}$ must be between 0 and 1 . The secondary circumplex is obtained by replacing $x$ and $y$ in Equation 1 by $x_{p_{x}}^{*}$ and $y_{p_{y}}^{*}$ computed in Equations 2 and 3:

$$
x_{p_{x}}^{*}=\sqrt{p_{x}} \bullet x+\sqrt{1-p_{x}} \bullet u
$$$$
y_{p_{y}}{ }^{*}=\sqrt{p_{y}} \bullet y+\sqrt{1-p_{y}} \bullet v
$$

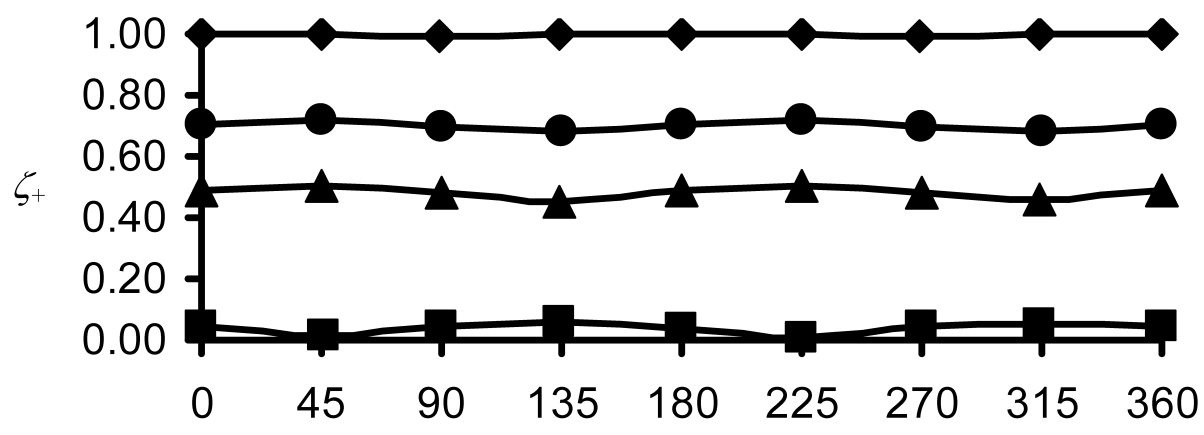

Location $\left(\theta_{i}\right)$ in secondary circumplex

\section{Figure A1}

$\zeta_{+}$for each variable in the secondary circumplex: Base and secondary circumplexes share two axes. Overlap is $100 \%$ (indicated by $\diamond$ ), $50 \%$ (indicated by $\bullet$ ), 25\% (indicated by $\mathbf{\Delta}$ ), and $0 \%$ (indicated by $\mathbf{\square}$ ). 
$C_{3}$ and $C_{4}$ were formed to represent $50 \%$ and $25 \%$ overlap respectively. $C_{3}$ was formed in a way identical to the procedure used to form $C_{2}$, except that $\sqrt{.50} \bullet x+\sqrt{.50} \bullet u$ replaced $x$, and $\sqrt{.50} \bullet y+\sqrt{.50} \bullet v$ replaced $y$. ( $x$ and $y$ were the random variates used to form the base circumplex.) To each of these 8 , we added a separate "random error" variable. $C_{4}$ was formed in a way identical to that for $C_{2}$ except that $x$ was replaced with $\sqrt{.25} \bullet x+\sqrt{.75} \bullet u$ and $y$ replaced $\sqrt{.25} \bullet y+\sqrt{.75} \bullet v$. To each of these 8 we added a separate "random error" variable.

Variables from $C_{3}$ and $C_{4}$ were located on the circumference of the base circumplex in the same manner as in the preceding simulations. Results are given in Figure A1. The second straight line in Figure A1 (composed of $\mathbf{0}$ ) shows the $\zeta_{+}$for the variables of $C_{3}$. $\zeta_{+}$ranged from .68 to .72 . The square of the $\zeta_{+}$values shows that, on average, $49 \%$ of variance of the secondary variables was explained by the base circumplex model. This empirical figure agrees well with the prescribed degree of overlap between $C_{3}$ and the base $-50 \%$. The third straight line in Figure A1 (composed of $\mathbf{\Delta}$ ) shows the $\zeta_{+}$ for the variables of $C_{4}$. $\zeta_{+}$ranged from .46 to .50 . On average, $23 \%$ of variance of the secondary variables was explained by the base circumplex model. This empirical figure agrees well with the prescribed degree of overlap between $C_{4}$ and the base $-25 \%$.

Overlap on the Vertical Axis of the Base Circumplex. We now consider the case when the secondary circumplex overlaps the base circumplex along one axis only. We begin by creating a secondary circumplex $\left(C_{5}\right)$ whose vertical axis was identical to, and whose horizontal axis was independent of, that of the base circumplex. $C_{5}$ was formed from $u$ and $y$ where $y$ was found in the base. We created 8 variables using Equation 1, each a linear combination of $u$ and $y$. To each of these 8 , we added a separate "random error" variable.

From the base circumplex and the secondary circumplex formed in this way, we had a total of 20 variables. For these, we sampled 1000 cases. We then used CIRCUM-extension to add to the base circumplex each of the 8 variables of the secondary circumplex, one at a time. Figure A2 (the topmost pattern composed of $\bullet$ ) shows the $\zeta_{+}$for each of the 8 , plotted as a function of the variable's angle $\left(\theta_{i}\right)$ within the secondary. $\zeta_{+}$ranged from .05 to 1.00 . The resulting pattern is no longer a straight line. The $\zeta_{+}$values are negligible at $0^{\circ}$ and $180^{\circ}$ but are 1.00 at $90^{\circ}$ and $270^{\circ}$. Moderate values are found at the remaining angles. For the variables with negligible $\zeta_{+}$, the $\theta_{+}$are meaningless. For others, $\theta_{+}$are extremely helpful in interpreting the results here. Variables with the $\zeta_{+}$value of $1.00\left(90^{\circ}\right.$ and $\left.270^{\circ}\right)$ shows where $C_{5}$ intersects with the 
vertical axis of the base circumplex, which was the prescribed location and degree of overlap between $C_{5}$ and the base.

We next turn to secondary circumplexes bearing smaller degrees of overlap with the vertical axis of the base. Relying on Equations 2 and 3, we formed $C_{6}$ and $C_{7}$ to represent $50 \%$ and $25 \%$ overlap. $C_{6}$ was formed in a way identical to that for $C_{5}$ except that $\sqrt{.50} \bullet y+\sqrt{.50} \bullet v$ replaced $y$. To each of these 8 we added a separate "random error" variable. $C_{7}$ was formed in a way identical to that for $C_{5}$ except that $\sqrt{.25} \bullet y+\sqrt{.75} \bullet v$ replaced $y$. To each of these 8 we added a separate "random error" variable.

Variables from $C_{6}$ and $C_{7}$ were located on the circumference of the base circumplex in the same manner as in the preceding simulations. Results are given in Figure A2. The second line in Figure A2 (composed of 0 ) shows the $\zeta_{+}$for each of the 8 variables of $C_{6}$. $\zeta_{+}$ranged from .04 to .69. Again, the $\zeta_{+}$values are negligible at $0^{\circ}$ and $180^{\circ}$ and highest at $90^{\circ}$ and $270^{\circ}$. Moderate values are found at the remaining angles. Variables with the $\zeta_{+}$ value of $.69\left(90^{\circ}\right.$ and $\left.270^{\circ}\right)$ shows where $C_{6}$ yields $48 \%$ of overlap on the vertical overlap axis of the base circumplex. The third line in Figure A2 (composed of $\mathbf{\Lambda}$ ) shows the $\zeta_{+}$for each of the 8 variables of $C_{7} . \zeta_{+}$ranged from .04 to .48 . Again, the $\zeta_{+}$values are negligible at $0^{\circ}$ and $180^{\circ}$ but highest at $90^{\circ}$ and $270^{\circ}$. Intermediate values are found at the remaining angles.

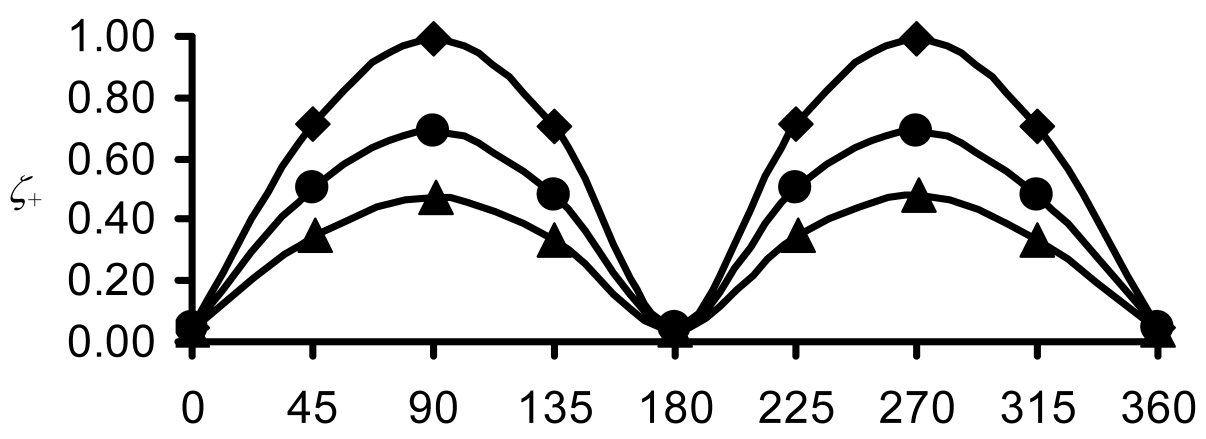

Location $\left(\theta_{i}\right)$ in secondary circumplex

\section{Figure A2}

$\zeta_{+}$for each variable in the secondary circumplex: Base and secondary circumplexes share the axis at $0^{\circ}-180^{\circ}$. Overlap is $100 \%$ (indicated by $\diamond$ ), $50 \%$ (indicated by $\bullet$ ), and $25 \%$ (indicated by $\boldsymbol{\Lambda})$. 
Variables with the $\zeta_{+}$values of .47 and $.48\left(90^{\circ}\right.$ and $\left.270^{\circ}\right)$ shows where $C_{7}$ yields $23 \%$ of overlap on the vertical axis of the base circumplex.

Overlap on the Horizontal Axis of the Base Circumplex. In parallel fashion, we examine a different axis of overlap between base and secondary circumplexes. We again begin by creating a secondary circumplex $\left(C_{8}\right)$ with one identical axis with the base circumplex (in this case, the horizontal axis). $C_{8}$ was formed from $x$ and $v$. We created 8 variables using Equation 1, each a linear combination of $x$ and $v$. To each of these 8 we added a separate "random error" variable. We then repeated the simulation procedures. Figure A3 (the topmost line composed of $\bullet$ ) shows the $\zeta_{+}$for each of the 8 , plotted as a function of the variable's angle $\left(\theta_{i}\right)$ within the secondary. $\zeta_{+}$ ranged from .04 to 1.00 . The $\zeta_{+}$values are negligible at $90^{\circ}$ and $270^{\circ}$ but are 1.00 highest at $0^{\circ}$ and $180^{\circ}$. Moderate values are found at the remaining angles. Variables with the $\theta_{+}$value of $1.00\left(0^{\circ}\right.$ and $\left.180^{\circ}\right)$ show that the horizontal axis of $C_{8}$ intersects entirely with that of the base circumplex the prescribed degree of overlap between $C_{8}$ and the base.

We formed $C_{9}$ and $C_{10}$ to represent $50 \%$ and $25 \%$ overlap. $C_{9}$ was formed in a way identical to that for $C_{8}$ except that $\sqrt{.50} \bullet x+\sqrt{.50} \bullet u$ replaced $x$. To each of these 8 we added a separate "random error"

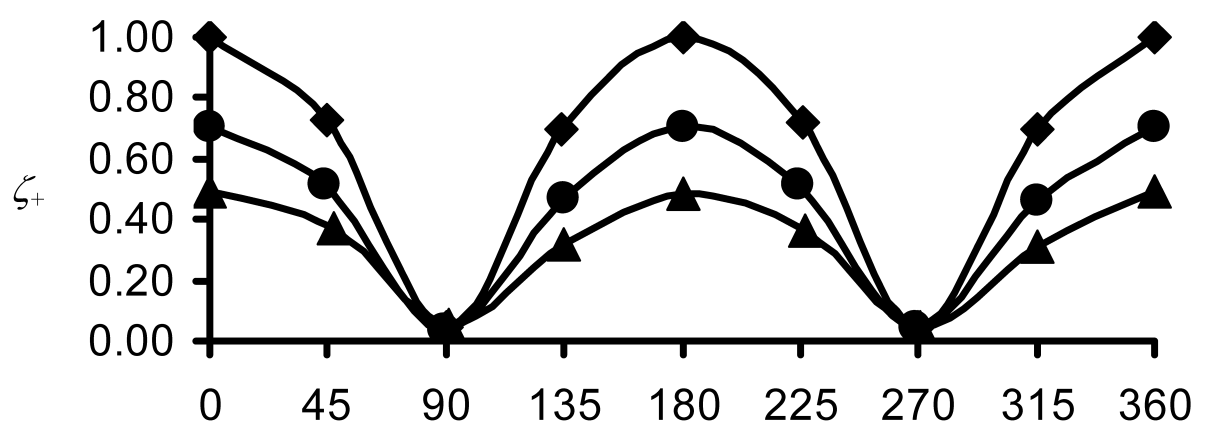

Location $\left(\theta_{i}\right)$ in secondary circumplex

\section{Figure A3}

$\zeta_{+}$for each variable in the secondary circumplex: Base and secondary circumplexes share the axis at $90^{\circ}-270^{\circ}$. Overlap is $100 \%$ (indicated by ), $50 \%$ (indicated by ), and $25 \%$ (indicated by $\mathbf{\Delta})$. 
variable. $C_{10}$ was formed in a way identical to that for $C_{8}$ except that $\sqrt{.25} \bullet x+\sqrt{.75} \bullet u$ replaced $x$. To each of these 8 we added a separate "random error" variable.

Variables from $C_{9}$ and $C_{10}$ were located on the circumference of the base circumplex in the same manner as in the preceding simulations. Results are given in Figure A3. The second line in Figure A3 (composed of ) shows the $\zeta_{+}$for each of the 8 variables of $C_{9}$. $\zeta_{+}$ranged from .04 to .70 . $\zeta_{+}$values are negligible at $90^{\circ}$ and $270^{\circ}$ but highest at $0^{\circ}$ and $180^{\circ}$. Variables with the $\zeta_{+}$ value of $.70\left(0^{\circ}\right.$ and $\left.180^{\circ}\right)$ show that the horizontal axis of $C_{9}$ yields $49 \%$ of overlap with that of $C_{8}$. The third line in Figure A3 (composed of m) shows that $\zeta_{+}$for each of the 8 variables of $C_{10}$. $\zeta_{+}$ranged from .05 to .49. Again, the $\zeta_{+}$values are negligible at $90^{\circ}$ and $270^{\circ}$ but highest at $0^{\circ}$ and $180^{\circ}$. Intermediate values are found at the remaining angles. Variables with the $\zeta_{+}$ values of .49 and $.48\left(90^{\circ}\right.$ and $\left.270^{\circ}\right)$ shows that the horizontal axis of $C_{10}$ yields $24 \%$ of overlap with that of $C_{8}$, close to the prescribed $25 \%$ degree of overlap. In short, the results for $C_{8}$ through $C_{10}$ (overlap on the horizontal axis) replicate the results for $C_{5}$ through $C_{7}$ (overlap on the vertical axis).

Summary. These simulations show that application of CIRCUMextension can reveal how and where two circumplexes are related. $\zeta_{+}$ estimates the degree of relation and the $\theta_{+}$estimates the location of the overlap. Small non-zero values of $\zeta_{+}$need to be ignored. When all variables show a negligible $\zeta_{+}$, the two circumplexes are unrelated. When all $\zeta_{+}$are substantial, then the two circumplexes are related along both axes (indeed, they might be the same structure), with $\theta_{+}$estimating the rotation needed to align them. When $\zeta_{+}$are mixed but of the "two humps" pattern of Figures $\mathrm{A} 2$ and $\mathrm{A} 3$, then the two circumplexes overlap along a single axis. The degree of relation is estimated by variables with the highest $\zeta_{+}$, and the axis of overlap estimated by an average $\theta_{+}$from the cluster of adjacent variables with non-negligible $\zeta_{+}$.

It should be straightforward to extrapolate these results to cases where the angle and degree of overlap is other than those used here. The empirical case reported in the body of this article shows that the patterns found in the simulations can also be found in empirical data. Altogether, these informal and preliminary results suggest that CIRCUM-extension could be developed formally into a method for charting the relationship between two circumplexes of the sort assumed here. It might be less straightforward, however, to generalize to the class of all circumplexes. 